\title{
From Thomson Formula to Resonant Equivalent Diagrams
}

\author{
L. Tomawski ${ }^{a, *}$, I. MrÓz ${ }^{b}$ And Z. KukUŁA ${ }^{c}$ \\ ${ }^{a}$ University of Silesia, August Chełkowski Institute of Physics, \\ 75 Putku Piechoty 1A, 41-500 Chorzów \\ ${ }^{b}$ University of Wrockaw, Institute of Experimental Physics, \\ pl. M. Borna 9, 50-204 Wrockaw, Poland \\ ${ }^{c}$ University of Silesia, Institute of Computer Science, \\ 75 Pułku Piechoty 1A, 41-500 Chorzów, Poland \\ Doi: $10.12693 /$ APhysPolA.139.304 \\ *e-mail: tomawski.l@gmail.com
}

\begin{abstract}
Until the 1970s, only three basic elements: R, C, and L have been used to construct RLC circuits. These elements are two-ports of zero order (resistances) and of first order (capacitances and inductances). Their presence in electronic circuits causes a certain phase shift between voltage and current. In 1969, Bruton published an inductorless filter. There are two two-ports of second order (supercapacitances) in this circuit. Supercapacitance causes a phase shift of 180 degrees. In the next years, many two-ports of higher orders (e.g. of second and third order) were developed and, in theory, their number may be infinite. The use of such two-ports in resonant circuits makes it possible to obtain other phase shifts. In this paper, we show how to transform basic circuits into their resonant equivalent diagram. The analysis of these diagrams allows us to describe precisely resonant phenomena occurring in the circuit and to identify all resonant circuits, even when the basic diagram does not indicate resonance properties.
\end{abstract}

topics: basic blocks of electronics, FDNR, FDNC, FDNCap

\section{Introduction}

Resonance phenomena are identified in many areas of science and technology. For oscillating systems, "Repeated disturbances can increase the amplitude of the oscillations if they are applied in synchrony with the natural frequency. Even a very small disturbance, repeated periodically at just the right frequency, can cause a very large amplitude motion to build up" [1]. The effect of increasing amplitude is illustrated by a universal resonant curve. The shape of this curve is determined by a quality factor that can be calculated using a suitable formula. In the field of electricity, a formula for the resonant frequency of an LC resonant circuit was derived by William Thomson (1824-1907). Understanding of resonance phenomena in electric circuits has evolved. Initially, they were analyzed in electrotechnics, and, later, in electronics and circuit theory.

Referring to [2], in this paper we present resonant equivalent diagrams that are derived for various resonant networks. These diagrams are composed of two-ports of various orders. In theory, two-ports can be of orders from 0 to infinity. Two-ports of third order were described, e.g., in [3]. We show that the presentation of resonance phenomena using resonant equivalent diagrams provides more information than using conventional basic diagrams.

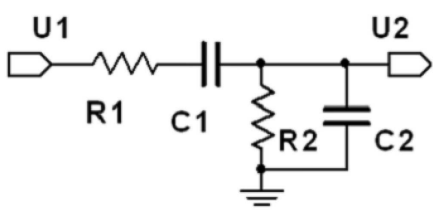

Fig. 1. The Wien circuit.

In 1891, Max Wien (1866-1938) published an RC circuit [4], named the Wien circuit (the Wien bridge), shown in Fig. 1.

The frequency response of this circuit has a resonant nature. However, since inductance does not appear in the circuit, the frequency of the Wien circuit was called "pseudo-resonant" and the quality factor was not specified. In those years, any resonant circuits different than an RCL circuit were not known.

The situation changed in 1969, when Leonard Bruton published an inductorless filter [5] which was a transformed RLC filter. As a result of transformation, resonance phenomena observed in the basic filter occurred between conductances and negative conductances of second order in the transformed filter. Therefore, a resonant circuit was created. In this circuit, active elements (conductances) resonate. A suitable transformation is shown in Fig. $2 \mathrm{a}-\mathrm{c}$. 

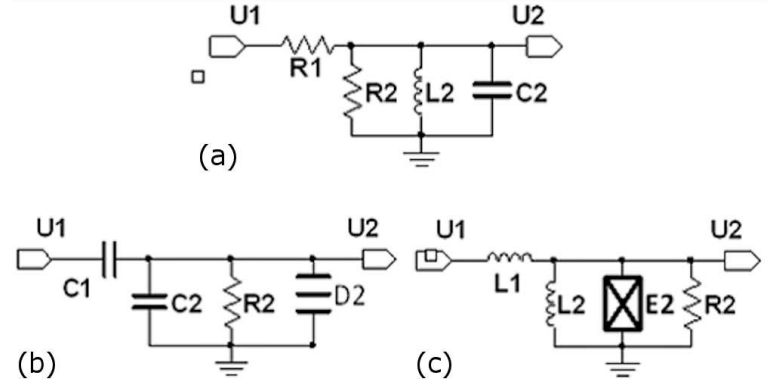

Fig. 2. A filter with the RLC circuit (a), with the DCG circuit (b) and with the ELR circuit (c).

The filter shown in Fig. 2a is the basic circuit for analysis. Its transmittance can be calculated as

$$
\frac{U_{2}}{U_{1}}=\frac{\frac{1}{R_{1}}}{\frac{1}{R_{1}}+\frac{1}{R_{2}}+\frac{1}{s L_{2}}+s C_{2}} .
$$

The right side of (1) is the ratio of the admittances of suitable two-ports and it is unitless. By multiplying the nominator and denominator of (1) by the operator $s$ we obtain

$$
\frac{U_{2}}{U_{1}}=\frac{s C_{1}}{s C_{1}+s C_{2}+\frac{1}{R_{2}}+s^{2} D_{2}} .
$$

Let us construct a scheme presented in Fig. 2b. From the comparison of Fig. $2 a$ and $b$, it can be concluded that during the process of transformation the resistances $R_{1}$ and $R_{2}$ are replaced by the capacitances $C_{1}$ and $C_{2}$, the inductance $L_{2}$ is replaced by the resistance $R_{2}$ and the capacitance $C_{2}$ is replaced by the supercapacitance $D_{2}$.

When the nominator and denominator of (1) are multiplied by $1 / s$, we obtain a circuit presented in Fig. $2 \mathrm{c}-$ the resistances $R_{1}$ and $R_{2}$ are replaced by the inductances $L_{1}$ and $L_{2}$, the capacitance $C_{2}$ is replaced by the resistance $R_{2}$ and the inductance $L_{2}$ is replaced by the superinductance $E_{2}$. The transmittance of the circuit shown in Fig. 2c is given by

$$
\frac{U_{2}}{U_{1}}=\frac{\frac{1}{s L_{1}}}{\frac{1}{s L_{1}}+\frac{1}{s L_{2}}+\frac{1}{s^{2} E_{2}}+\frac{1}{R_{2}}} .
$$

In Fig. 2b and c, two new two-ports can be observed: supercapacitance — frequency dependent negative conductance (FDNC) and superinductance - frequency dependent negative resistance (FDNR) that are active elements of second order. FDNC is a frequency-dependent negative conductance, its admittance is negative: $-\omega^{2} D\left[\mathrm{~A} \mathrm{~s} \mathrm{~s}^{2} / \mathrm{V}\right]$ and FDNR is a frequency-dependent negative resistance and its impedance is also negative: $-\omega^{2} E\left[\mathrm{~V} \mathrm{~s}^{2} / \mathrm{A}\right]$. These elements can be produced only electronically.

Since the nominator and denominator of the transmittance (1) of the circuit in Fig. 2a can by multiplied by $s^{n}$ or $1 / s^{n}$, where $n$ is arbitrarily large, in theory a number of two-ports of higher orders may also be arbitrarily large. In practice, $n$ is not greater than 4 or, maximum, 5 .
Three variants of a 2RLC network are analyzed below. There are two-ports of third (FDNCap) and fourth (FDPCond) order in their resonant equivalent diagrams. The names of these two-ports reflect their properties, i.e., FDNCap (frequency dependent negative capacitance) and FDPCond (frequency dependent positive conductance).

\section{Examples}

The example described above shows that the wellknown electronic circuits can be transformed into circuits containing suitably connected two-ports of various orders. Such resonant equivalent diagrams lead to a new method of testing resonant properties of analog circuits.

Figure 3 shows an RLC resonant circuit (a) transformed into its resonant equivalent diagram (c). The system in Fig. 3a can be presented as a voltage divider composed of admittances $\mathrm{Y}_{1}$ and $\mathrm{Y}_{2}$ (Fig. 3b). Then, from the comparison of the operator transmittances of the circuits presented in Fig. 3a and b, the resonant equivalent diagram can be constructed (Fig. 3c).

The operator transmittance of the RLC circuit (see Fig. 3a) can be expressed as

$$
T(s)=\frac{1 / R}{s^{2} L C / R+s C+1 / R} .
$$

The transmittance of the voltage divider (see Fig. 3b) is given by

$$
T(s)=\frac{Y_{1}}{Y_{1}+Y_{2}} .
$$

From (4) and (5) we obtain the admittances $Y_{1}$ and $Y_{2}$ as

$$
Y_{1}=\frac{1}{R}
$$

and

$$
Y_{2}=s^{2} L C / R+s C \text {. }
$$

The resonant equivalent diagram of the RLC circuit (Fig. 3c) can be obtained after substitution $s=\mathrm{i} \omega$. The admittance $\mathrm{Y}_{2}$ is composed of connected in parallel: capacitance $\mathrm{C}$ and FDNC
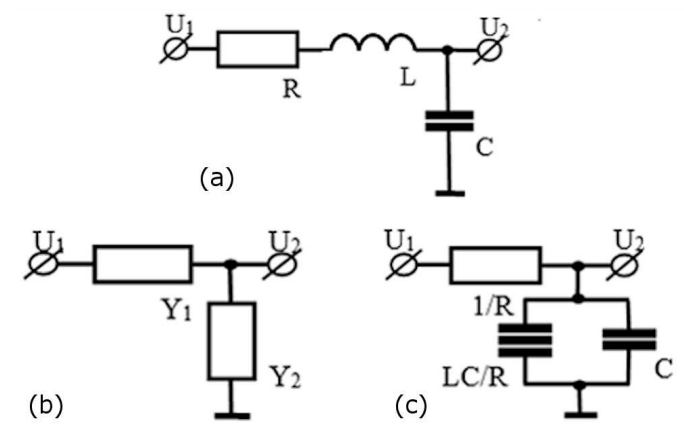

Fig. 3. The example of an RLC resonant circuit (a), the voltage divider (b) and the resonant equivalent diagram (c). 


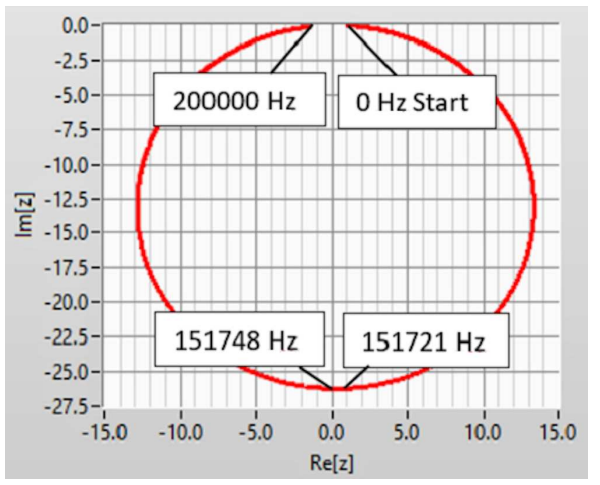

Fig. 4. Characteristics of the resonant equivalent diagram (Fig. 3c) on the complex plane.

(a negative conductance dependent on the angular frequency, i.e., $\left.-\omega^{2} L C / R\right)$. The admittance $\mathrm{Y}_{1}$ is conductance of zero order.

Characteristics on the complex plane of the resonant equivalent diagram (Fig. 3c) are shown in Fig. 4. They were obtained by substituting $s=\mathrm{i} \omega$ into (4) (the calculations were performed for $R=40 \Omega, L=1.1 \mathrm{mH}$ and $C=1 \mathrm{nF}$ to relate to the experimental results presented in [2]). The plot was prepared using the Math Script using Riemann zeta.vi procedure, LabVIEW 2017 computer software (the same procedure was used to prepare Figs. 6, 8, 10 and 12).

The plot in Fig. 4 starts at $(1, \mathrm{i} 0)$ and ends at $(0, \mathrm{i} 0)$. The last point would be achieved for infinite frequency. The resonant frequency $151.748 \mathrm{kHz}$ calculated from the simplified Thomson formula $f=$ $1 / 2 \pi \sqrt{L C}$ corresponds to the point $(0, \mathrm{i} 26.2)$. Taking into account losses caused by $40 \Omega$ resistance, we obtain the resonant frequency equal to $151.721 \mathrm{kHz}$. This value is calculated using

$$
f=\frac{1}{2 \pi} \sqrt{\frac{1}{L C}-\frac{R^{2}}{4 L^{2}}} .
$$

Suitability of using resonant equivalent diagrams for analyzing resonant phenomena in analog electronic circuits is illustrated below. We consider several variants of networks composed of suitably connected circuits shown in Fig. 3a.

Two connected circuits presented in Fig. 3a, called the 2RLC network, are shown in Fig. 5a. The resonant equivalent diagram, obtained using transformations similar to these presented in Fig. 3b and $\mathrm{c}$, is shown in Fig. 5b.

The resonant equivalent diagram is more composed than the basic diagram of the network. Namely, in the resonant equivalent diagram we observe six different elements, while in the basic network we have only three different elements. Therefore, the information provided by the resonant equivalent diagram is richer. In the scheme shown in Fig. 5b, we see five pairs of elements, for which spectral admittances differ in signs. Moreover, the ratios of these admittances contain factors $\omega^{2}$ or $1 / \omega^{2}$. These pairs of two-ports create

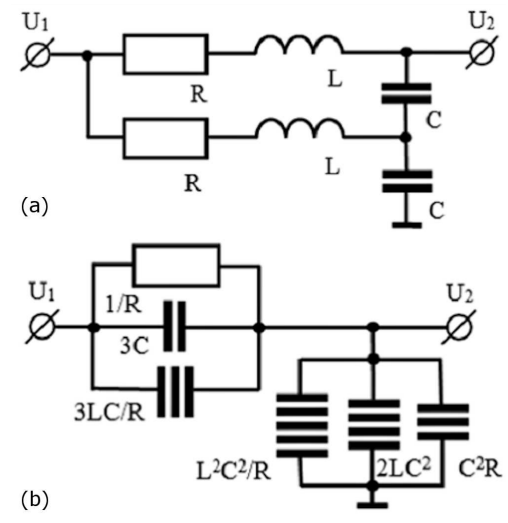

Fig. 5. The 2RLC network (a) and its resonant equivalent diagram (b).

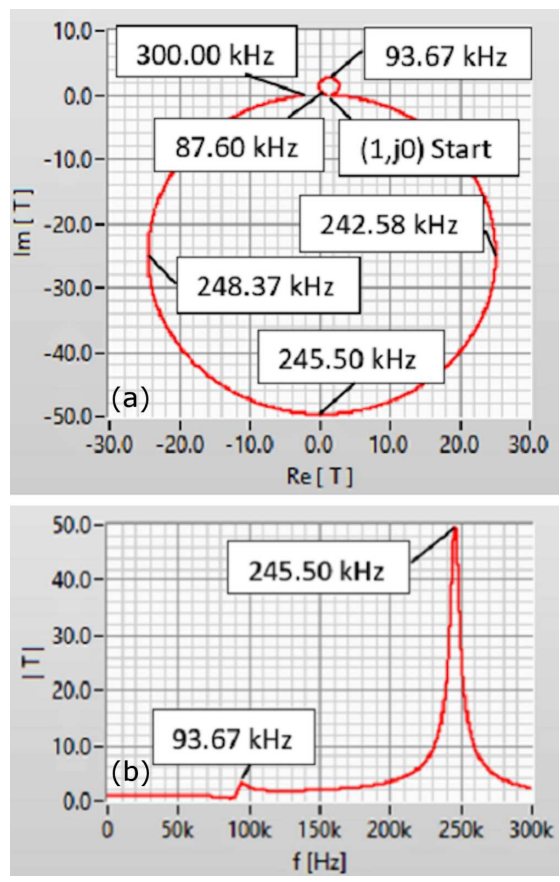

Fig. 6. Characteristics of the 2RLC network on the complex plane (a) and a modulus of the transmittance of the 2RLC network (b).

resonant circuits connected in series or in parallel, and the resonant frequencies of the circuits depend on the values of the RLC elements. Pairs of the resonating two-ports, the corresponding oscillation equations and the resonant frequencies are listed in Table I.

The resonant frequencies shown in the last column are calculated using the oscillation equations given in Table I. The values of frequencies shown in Fig. 6a may slightly differ from the values shown in Table I as long as the considered resonant frequencies have close values. The details can be analyzed using the frequency dependence of the modulus of the transmittance of the network. For the 2RLC network, the frequency dependence of the modulus of the transmittance is shown in Fig. $6 \mathrm{~b}$. 
Resonant circuits in the 2RLC network.

TABLE I

\begin{tabular}{c|l|c|c}
\hline \hline $\begin{array}{c}\text { Resonating } \\
\text { admittance two-ports }\end{array}$ & \multicolumn{1}{|c|}{ Resonance } & $\begin{array}{c}\text { Oscillation } \\
\text { equation }\end{array}$ & $\begin{array}{c}\text { Resonant } \\
\text { frequency }\end{array}$ \\
\hline$\frac{3 L C}{R}, \frac{1}{R}$ & conductive, parallel, in the horizontal branch & $\mathrm{i}^{2} \omega^{2} \frac{3 L C}{R}+\frac{1}{R}=0$ & $87.6 \mathrm{kHz}$ \\
$C^{2} R, \frac{1}{R}$ & conductive, serial & $\mathrm{i}^{2} \omega^{2} C^{2} R+\frac{1}{R}=0$ & $3979 \mathrm{kHz}$ \\
$2 L C^{2}, 3 C$ & capacitive, serial & $\mathrm{i}^{3} \omega^{3} 2 L C^{2}+\mathrm{i} \omega 3 C=0$ & $185.9 \mathrm{kHz}$ \\
$\frac{L^{2} C^{2}}{R}, \frac{3 L C}{R}$ & conductive, serial & $\mathrm{i}^{4} \omega^{4} \frac{L^{2} C^{2}}{R}+\mathrm{i}^{2} \omega^{2} \frac{3 L C}{R}=0$ & $262.8 \mathrm{kHz}$ \\
$\frac{L^{2} C^{2}}{R}, C^{2} R$ & conductive, parallel, in the vertical branch & $\mathrm{i}^{4} \omega^{4} \frac{L^{2} C^{2}}{R}+\mathrm{i}^{2} \omega^{2} C^{2} R=0$ & $5.79 \mathrm{kHz}$
\end{tabular}

Resonant circuits in the 2LCR network.

TABLE II

\begin{tabular}{c|l|c|c}
\hline \hline $\begin{array}{c}\text { Resonating } \\
\text { admittance two-ports }\end{array}$ & \multicolumn{1}{|c|}{ Resonance } & $\begin{array}{c}\text { Oscillation } \\
\text { equation }\end{array}$ & $\begin{array}{c}\text { Resonant } \\
\text { frequency }\end{array}$ \\
\hline$\frac{L^{2} C^{2}}{R}, C^{2} R$ & conductive, serial & $\mathrm{i}^{4} \omega^{4} \frac{L^{2} C^{2}}{R}+\mathrm{i}^{2} \omega^{2} C^{2} R=0$ & $5.79 \mathrm{kHz}$ \\
$\frac{2 L C}{R}, \frac{1}{R}$ & conductive, parallel, in the vertical branch & $\mathrm{i}^{2} \omega^{2} \frac{2 L C}{R}+\frac{1}{R}=0$ & $107.3 \mathrm{kHz}$ \\
$3 L C^{2}, 3 C$ & capacitive, parallel, in the vertical branch & $\mathrm{i}^{3} \omega^{3} 3 L C^{2}+\mathrm{i} \omega 3 C=0$ & $151.75 \mathrm{kHz}$ \\
$\frac{L^{2} C^{2}}{R}, \frac{2 L C}{R}$ & conductive, parallel, in the vertical branch & $\mathrm{i}^{4} \omega^{4} \frac{L^{2} C^{2}}{R}+\mathrm{i}^{2} \omega^{2} \frac{2 L C}{R}=0$ & $214.6 \mathrm{kHz}$ \\
$C^{2} R, \frac{1}{R}$ & conductive, serial & $\mathrm{i}^{2} \omega^{2} C^{2} R+\frac{1}{R}=0$ & $3978 \mathrm{kHz}$
\end{tabular}

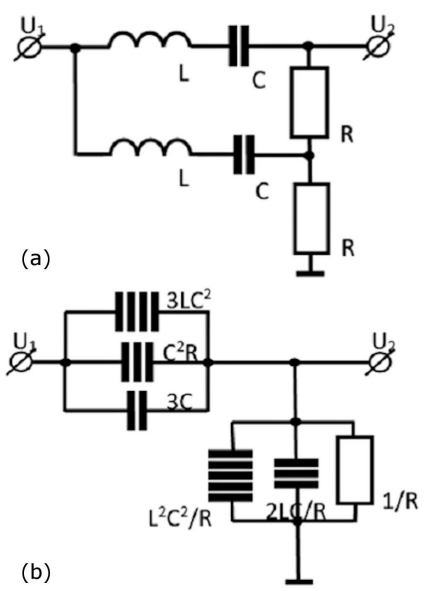

Fig. 7. The 2LCR network: a basic diagram (a) and its resonant equivalent diagram (b).

$\mathrm{R}, \mathrm{L}$, and C elements $(R=40 \Omega, L=1.1 \mathrm{mH}$ and $C=1 \mathrm{nF}$ ) can be connected in a $2 \mathrm{LCR}$ network shown in Fig. 7a. Its resonant equivalent diagram is presented in Fig. 7b.

The resonant equivalent diagram is composed of five pairs of two-ports that create resonant circuits. These circuits, the corresponding oscillation equations and resonant frequencies are listed in Table II.

Characteristics of the 2LCR network on the complex plane are shown in Fig. 8a, and the frequency dependence of the transmittance modulus is presented in Fig. 8b.

The third variant: the 2RCL network is presented in Fig. 9a, while its resonant equivalent diagram is shown in Fig. 9b.

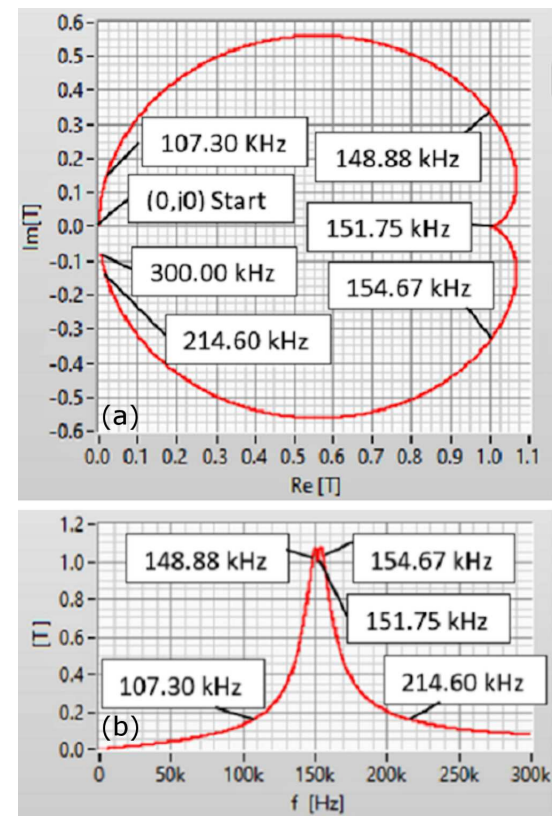

Fig. 8. Characteristics of the 2LCR network on the complex plane (a) and the transmittance modulus $z(i \omega)$ of the 2LCR network (b).

Five resonant circuits occurring in the resonant equivalent diagram of the 2RCL network, the corresponding oscillation equations and the resonant frequencies are shown in Table III.

The values of the resonant frequencies listed in Table III can be compared with the values shown in the characteristic of the network on the complex plane (Fig. 10a). The frequency dependence of the transmittance modulus of this network is shown in Fig. 10b. 
Resonant circuits in the 2RCL network.

TABLE III

\begin{tabular}{c|l|c|c}
\hline \hline $\begin{array}{c}\text { Resonating } \\
\text { admittance two-ports }\end{array}$ & \multicolumn{1}{|c|}{ Resonance } & $\begin{array}{c}\text { Oscillation } \\
\text { equation }\end{array}$ & $\begin{array}{c}\text { Resonant } \\
\text { frequency }\end{array}$ \\
\hline$\frac{3 L C}{R}, \frac{1}{R}$ & conductive, serial & $\mathrm{i}^{2} \omega^{2} \frac{3 L C}{R}+\frac{1}{R}=0$ & $87.6 \mathrm{kHz}$ \\
$C^{2} R, \frac{1}{R}$ & conductive, parallel, in the vertical branch & $\mathrm{i}^{2} \omega^{2} C^{2} R+\frac{1}{R}=0$ & $3979 \mathrm{kHz}$ \\
$3 L C^{2}, 2 C$ & capacitive, serial & $\mathrm{i}^{3} \omega^{3} 3 L C^{2}+\mathrm{i} \omega 2 C=0$ & $123.9 \mathrm{kHz}$ \\
$\frac{L^{2} C^{2}}{R}, \frac{3 L C}{R}$ & conductive, parallel, in the horizontal branch & $\mathrm{i}^{4} \omega^{4} \frac{L^{2} C^{2}}{R}+\mathrm{i}^{2} \omega^{2} \frac{3 L C}{R}=0$ & $262.8 \mathrm{kHz}$ \\
$\frac{L^{2} C^{2}}{R}, C^{2} R$ & conductive, serial & $\mathrm{i}^{4} \omega^{4} \frac{L^{2} C^{2}}{R}+\mathrm{i}^{2} \omega^{2} C^{2} R=0$ & $5.79 \mathrm{kHz}$
\end{tabular}

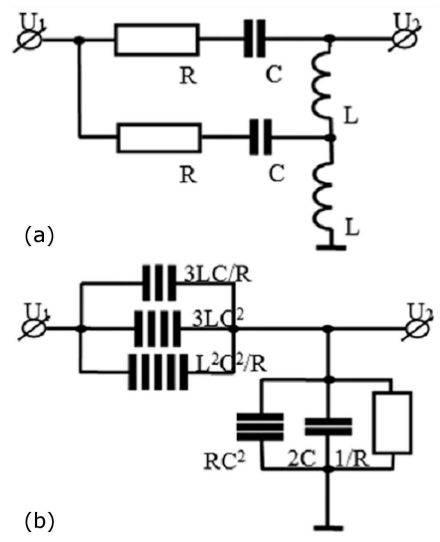

Fig. 9. The 2RCL network: a basic diagram (a) and its resonant equivalent diagram (b).

Comparison of the numerical values obtained for the three variants of networks described above lets us summarize common features and differences.

Common features:

1. The resonant equivalent diagrams of every network contain five different resonant circuits.

2. All resonant equivalent diagrams contain twoports of orders from zero to fourth. Moreover, two of six two-ports are of second order.

3. Great values (peaks) of the frequencydependent moduli are associated with big circles of the characteristics of the networks on the complex plane.

Differences:

1. In the 2RLC and 2RCL networks, the frequency $151.75 \mathrm{kHz}$ is not observed. This frequency is a resonant frequency of a single LC circuit. This value is present in the set of natural frequencies of the 2LCR network.

2. The dominant frequency of the $2 \mathrm{RLC}$ network $(245.5 \mathrm{kHz})$ is higher than the frequency of a single LC circuit $(151.75 \mathrm{kHz})$. In more complicated networks, e.g., 3RLC, 4RLC, etc., the dominant frequency deviates more and more from the value of $151.75 \mathrm{kHz}$.

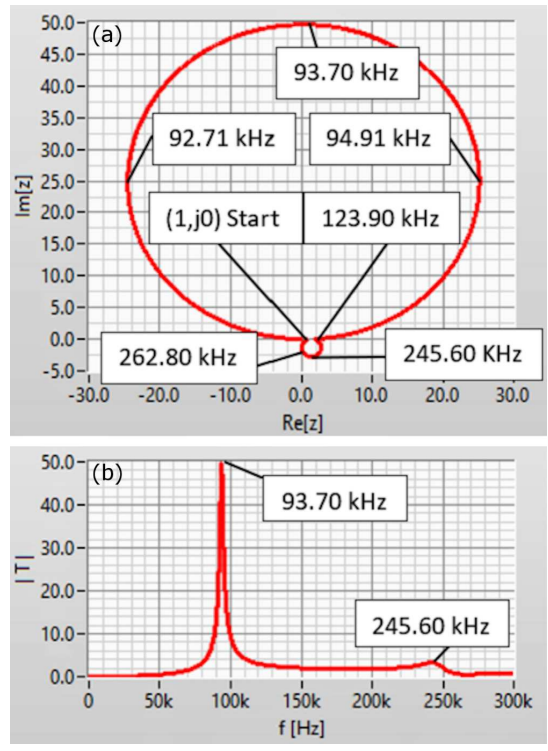

Fig. 10. Characteristics of the 2RCL network on the complex plane (a) and the transmittance modulus $z(\mathrm{i} \omega)$ of the 2RCL network (b).

3. The dominant frequency of the $2 \mathrm{RCL}$ network $(93.7 \mathrm{kHz})$ is smaller than the frequency of a single LC circuit $(151.75 \mathrm{kHz})$. In more complicated networks, e.g., 3RCL, 4RCL etc., the dominant frequency deviates more and more (towards lower values) from the value of $151.75 \mathrm{kHz}$.

Resonant equivalent diagrams are also useful for analyzing the Wien circuit (see Fig. 1). Its operator transmittance is given by

$$
T(s)=\frac{s C_{1}}{s^{2} C_{1} C_{2} R_{1}+s\left(C_{1}+C_{2}+C_{1} \frac{R_{1}}{R_{2}}\right)+\frac{1}{R_{2}}} .
$$

Transformations similar to these presented in Fig. 3 lead to the resonant equivalent diagram of the Wien circuit (Fig. 11b).

The resonant equivalent diagram (Fig. 11b) is more complex than the basic diagram. Although in both diagrams the same number of two-ports (four) can be identified, in the basic diagram only resistances of zero order and capacitances of first order are observed while in the equivalent resonant 


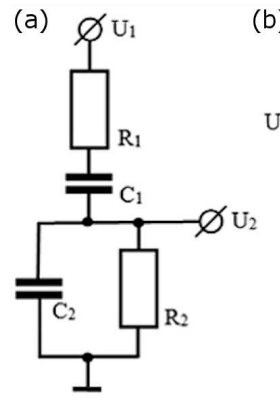

(b)

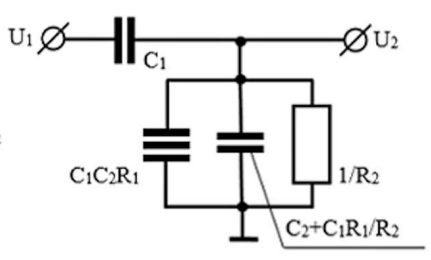

Fig. 11. The basic diagram of the Wien circuit (a) and its resonant equivalent diagram (b).

diagram we additionally have a two-port of second order (FDNC). If the values of $R_{1}$ and $R_{2}$ are equal and the values of $C_{1}$ and $C_{2}$ are equal, in the basic diagram we have only two two-ports, while in the resonant equivalent diagram four two-ports are present. Hence, it can be concluded that a resonant equivalent diagram is a better tool to describe phenomena occurring in the circuit than a basic diagram.

The resonant equivalent diagram of the Wien circuit (Fig. 11b) contains a resonant circuit. Conductive resonance occurs between the conductance $1 / R_{2}$ and the negative conductance $-\omega^{2} C_{1} C_{2} R_{1}$. The resonant frequency can be derived from

$$
-\omega^{2} C_{1} C_{2} R_{1}+\frac{1}{R_{2}}=0
$$

The solution of (10) leads to the Thomson formula

$$
\omega=\frac{1}{\sqrt{C_{1} C_{2} R_{1} R_{2}}} .
$$

After substituting $R_{1}=R_{2}=10 \mathrm{k} \Omega$ and $C_{1}=$ $C_{2}=1 \mathrm{nF}$, the value of the resonant frequency equals $15.915 \mathrm{kHz}$.

For $\omega$ given by (11) and $s=\mathrm{i} \omega$ substituted to (9) we obtain damping of the Wien circuit

$$
|T(\mathrm{i} \omega)|=\frac{1}{1+\frac{C_{2}}{C_{1}}+\frac{R_{1}}{R_{2}}} .
$$

For the above numerical data and the resonant frequency, the value of damping calculated according to $(12)$ is 0.3333 .

The capacitance $C_{2}+C_{1} R_{1} / R_{2}$ is a parasitic element of a conductive resonant circuit. Its value significantly influences the quality factor of the circuit. This quality factor can be expressed as a ratio of the admittance of one of the resonating vectors (e.g. $\left.1 / R_{2}\right)$ and the admittance of the parasitic vector $\omega\left(C_{2}+C_{1} R_{1} / R_{2}\right)$. Hence, for the quality factor one has

$$
Q=\frac{\sqrt{R_{1} C_{1} R_{2} C_{2}}}{C_{1} R_{1}+C_{2} R_{2}} .
$$

For $R_{1}=R_{2}=10 \mathrm{k} \Omega$ and $C_{1}=C_{2}=1 \mathrm{nF}$ we obtain $Q=0.5$.

The above equation describes the quality factor for the Wien circuit controlled from an infinite resistance source. For the circuit controlled from a low

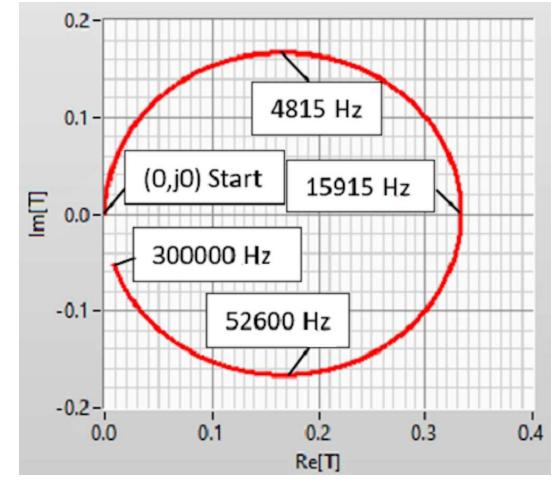

Fig. 12. Characteristics of the Wien circuit on the complex plane $\left(R_{1}=R_{2}=10 \mathrm{k} \Omega\right.$ and $C_{1}=$ $\left.C_{2}=1 \mathrm{nF}\right)$.

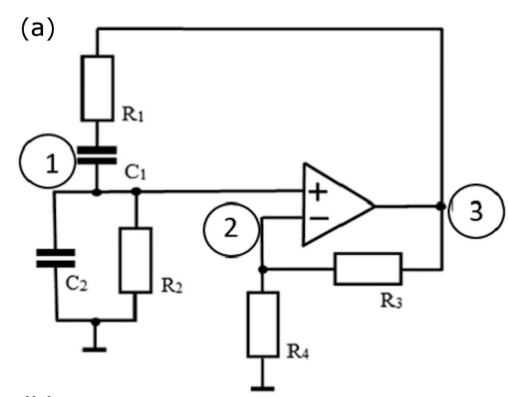

(b)

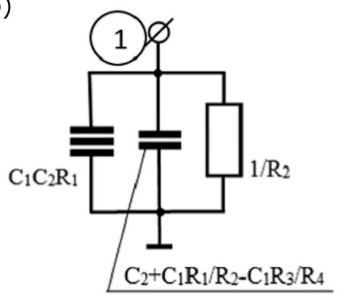

Fig. 13. The oscillator with the Wien circuit (a) and the two-port equivalent diagram of the admittances of nodes (1) or (2) (b).

resistance source, it should be assumed that $\mathrm{C}_{1}$ is connected in parallel to the remaining capacities and a quality factor can be expressed by

$$
Q=\frac{\sqrt{R_{1} C_{1} R_{2} C_{2}}}{C_{1}\left(R_{1}+R_{2}\right)+C_{2} R_{2}} .
$$

For $R_{1}=R_{2}=10 \mathrm{k} \Omega$ and $C_{1}=C_{2}=1 \mathrm{nF}$ we have $Q=0.3333$.

Figure 12 presents the characteristics of the Wien circuit on the complex plane (the transmittance (9) with substituted $s=\mathrm{i} \omega$ was used). The regular circle shows properties of the equivalent resonant diagram of the Wien circuit. The resonant frequency read from the characteristics (for $\operatorname{Im}\{T\}=0$ ) as well as calculated from (8) is $15915 \mathrm{~Hz}$. For this frequency, damping (read from the $\operatorname{Re}\{T\}$ axis) is $|T(\mathrm{i} \omega)|=0.333$. The same value can be obtained using (12).

A quality factor can be calculated from Fig. 12 as the ratio of the resonant frequency and the circuit bandwidth. In Fig. 12, two marked points, $4815 \mathrm{~Hz}$ 
and $52600 \mathrm{~Hz}$, determine the boundaries of the circuit band. The value of the quality factor calculated using this method is 0.333 .

The Wien circuit has been still used to construct a well-known oscillator (see, e.g., [6]) shown in Fig. 13.

The circles shown in Fig. 13 denote three nodes of the oscillator. Two of them (1 and 2 ) are free (since their voltages are not forced by the op amp outputs as it happens for the node (3)). Since the oscillator is not a transmission circuit, it is not possible to transform it into its resonant equivalent diagram using the procedure described above. However, it is possible to describe the phenomena in the oscillator by a natural vibration equation. This equation is expressed by admittance of a free node set to zero. After applying the classical node voltage method we obtain the following admittances of nodes 1 and 2 :

$$
\begin{aligned}
& Y_{1}=\frac{I}{U_{1}}=\frac{s^{2} R_{1} R_{2} C_{1} C_{2}+s\left(R_{1} C_{1}+R_{2} C_{2}-\frac{R_{2} R_{3}}{R_{4}} C_{1}\right)+1}{s R_{1} R_{2} C_{1}+R_{2}}, \\
& Y_{2}=\frac{I}{U_{2}}=\frac{-s^{2} R_{1} R_{2} C_{1} C_{2}-s\left(R_{1} C_{1}+R_{2} C_{2}-\frac{R_{2} R_{3}}{R_{4}} C_{1}\right)-1}{s R_{2} R_{3} C_{1}} .
\end{aligned}
$$

Setting (15) and (16) to zero leads to the oscillation equation of the oscillator

$$
\begin{aligned}
& s^{2} R_{1} R_{2} C_{1} C_{2} \\
& \quad+s\left(R_{1} C_{1}+R_{2} C_{2}-\frac{R_{2} R_{3}}{R_{4}} C_{1}\right)+1=0 .
\end{aligned}
$$

If $s=\mathrm{i} \omega$, it is possible to construct the resonant equivalent diagram shown in Fig. 13b. The quality factor can be given by

$$
Q=\frac{\sqrt{C_{1} C_{2} R_{1} R_{2}}}{R_{2}\left[C_{2}+C_{1}\left(\frac{R_{1}}{R_{2}}-\frac{R_{3}}{R_{4}}\right)\right]} .
$$

In (18), for $C_{1}=C_{2}=1 \mathrm{nF}, R_{1}=R_{2}=10 \mathrm{k} \Omega$, $R 3=20 \mathrm{k} \Omega, R_{4}=10.1 \mathrm{k} \Omega$ we obtain $Q=50$. This value has been checked using computer simulation (Multisim 14.0 package, AC method), and the result was 48 .

\section{Conclusions}

The considerations presented above show that resonant equivalent diagrams are very useful in analyzing resonant phenomena in electronic systems.

They provide more information than basic diagrams. Using them enables detailed analysis of resonances that are difficult to identify and describe, like in the Wien oscillator. It is worth stressing that in resonant equivalent diagrams, resonances are observed between "basic building blocks" — two-ports of various orders. This facilitates systematic classification of observed phenomena. We believe that resonant equivalent diagrams are really important for modern analog electronics and circuit theory.

\section{Acknowledgments}

The authors would like to thank National Instruments Poland Sp. z o.o. for their consent to use the LabVIEW 17.0 and Multisim 14.0 software.

\section{References}

[1] The New Encyclopedia Britannica, Vol. 24, 15th ed., 2002, p. 709.

[2] L. Tomawski, Z. Kukuła, Electron. World 2006, 26 (2006).

[3] S. Noguchi, Proc. IEEE ISCAS 1, 103 (1985).

[4] M. Wien, Ann. Phys. Chem. 280, 689 (1891).

[5] L.T. Bruton, IEEE Trans. Circuit Theory 16, 406 (1969).

[6] J. Punčochář in: 11th Int. Czech-Slovak Scientific Conf. Radioelektronika 2001, 2001. 